\title{
Oligoclonal Pattern/Abnormal Protein Bands in Post- Treatment Plasma Cell Myeloma Patients: Implications for Protein Electrophoresis and Serum Free Light Chain Assay Results
}

\author{
Gurmukh Singh
}

\begin{abstract}
Background: The impact of autologous stem cell transplantation (ASCT) in plasma cell myeloma patients on the frequency, quality, and timing of oligoclonal pattern in serum protein electrophoresis/immunofixation electrophoresis (SPEP/SIFE) and serum free light chain assay (SFLCA) was evaluated.
\end{abstract}

Methods: Laboratory results and clinical data for 251 patients with plasma cell myeloma, who had SPEP/SIFE and/or SFLCA performed between January 2010 and December 2016 were reviewed. The results for SPEP/SIFE and SFLCA were compared in patients with ASCT to those without ASCT. The implications of oligoclonal pattern in interpretation of SPEP/SIFE and SFLCA $-\kappa / \lambda$ ratio were addressed.

Results: In 251 patients, a total of 3,134 observations, of either SPEP/ SIFE and/or SFLCA, were reviewed. One hundred fifty-nine patients received ASCT. The incidence of oligoclonal patterns was significantly higher after ASCT. More than half of the oligoclonal patterns developed in the first year after transplantation. In 13 of the 84 patients with lambda chain restricted plasma cell myeloma, the $\kappa / \lambda$ ratio was kappa dominant in the presence of oligoclonal pattern. There was no reversal of $\kappa / \lambda$ ratio in patients with kappa chain restricted plasma cell myelomas.

Conclusions: ASCT is associated with significantly higher incidence of oligoclonal patterns than with chemotherapy alone. The presence of oligoclonal patterns has the potential to interfere with the interpretation of SPEP/SIFE and ascertainment of complete remission. At a minimum, the oligoclonal pattern caused an incorrect kappa dominant $\kappa / \lambda$ ratio in $15.5 \%$ of patients with lambda chain restricted plasma cell myeloma. If a similar rate were to be applied to the 167 kappa chain myeloma patients, about 26 of these would have displayed an erroneous kappa chain dominant $\kappa / \lambda$ ratio. The presence of oligoclonal

Manuscript submitted April 16, 2017, accepted May 2, 2017

Department of Pathology, Medical College of Georgia at Augusta University, 1120 15th Street, BI 2008A, Augusta, GA 30912, USA.

Email: gurmukhsinghmdphd@yahoo.com

doi: https://doi.org/10.14740/jocmr3049w pattern further degrades the performance of already dubious SFLCA. The need for recording the location of monoclonal spike in SPEP/ SIFE and higher resolution protein electrophoresis methods are highlighted.

Keywords: Serum protein electrophoresis; Oligoclonal pattern; Abnormal protein bands; Location of monoclonal spike; Serum free light chain assay; Complete remission; Multiple myeloma; Plasma cell myeloma

\section{Introduction}

Plasma cell myeloma/multiple myeloma is one of the most common hematologic malignancies in adults in the United States, and second in incidence only to non-Hodgkin lymphoma group of tumors $[1,2]$. These malignant lesions are generally treatable but mostly incurable. Myelomas are malignant clonal proliferations of plasma cells, terminally differentiated $\mathrm{B}$ cells, and are usually associated with circulating monoclonal immunoglobulins [3]. Generally, only an immunoglobulin consisting of one heavy chain type and one light chain type produces a sharp band in serum protein electrophoresis (SPEP) and serum immunofixation electrophoresis (SIFE). In about $80 \%$ of cases, an intact immunoglobulin constitutes the monoclonal protein, with $\operatorname{IgG}$ kappa being the most common type. In about $15-18 \%$ of the patients, only light chains are produced and a serum light chain band only may be detectable. However, in some cases, urine protein electrophoresis (UPEP) and urine immunofixation electrophoresis (UIFE) may be needed to identify a monoclonal light chain for the diagnosis. In a small number of patients, more than one clone of the malignant immunoglobulin may be present. In the case of biclonal myeloma, the biclonality may be due to different heavy chains or different light chains. Triclonal malignant immunoglobulins are observed more rarely. In about $1 \%$ of myelomas, the malignant cells fail to secrete any immunoglobulin or light chains, i.e. non-secretory myelomas [4-7].

SPEP/SIFE and UPEP/UIFE are the gold standard diagnostic tests for myeloma, along with bone marrow examination to assess the proportion of myeloma cells in marrow, clonality determination, and genetic changes in malignant cells $[8$, 
9]. Even non-secretory myelomas will usually exhibit intracellular expression of monoclonal immunoglobulins, or light chains.

Serum free light chain assay (SFLCA) has been promoted for the diagnosis and monitoring of proliferative disorders of plasma cells. The presence of monoclonal immunoglobulins or monoclonal light chain gammopathy often results in an excess of the relevant light chain in serum and an ensuing abnormal ratio of kappa to lambda light chain quantity in serum. The normal ratio of kappa to lambda chains in serum is $0.26-1.65$. Dominance of kappa or lambda light chains is taken to be an indication of monoclonal proliferation of the relevant immunoglobulin; though there are many exceptions to this rule, an abnormal $\kappa / \lambda$ ratio is not diagnostic of monoclonal gammopathy and a normal $\kappa / \lambda$ ratio does not exclude monoclonal gammopathy [10-12].

With the introduction of new chemotherapy and hematopoietic stem cell transplantation, the survival of myeloma patients has improved markedly $[4,13]$. One of the side effects of chemotherapy, particularly with bortezomib and thalidomide congeners, and especially following hematopoietic stem cell transplantation, is the development of oligoclonal pattern or abnormal protein bands in SPEP/SIFE [14]. The oligoclonal pattern has also been referred to as abnormal protein bands and may be a better term as only one band may be noted, in addition to the malignant immunoglobulin spike [15]. The development of oligoclonal pattern/abnormal protein band is associated with additional restricted heterogeneity immunoglobulins, with or without detectable primary malignant monoclonal immunoglobulin. The additional restricted heterogeneity immunoglobulins may be intact immunoglobulins or heavy or light chains only. IgG kappa restricted heterogeneity immunoglobulin appears to the most common element of oligoclonal response. Since IgG kappa is also the most common type of myeloma immunoglobulin, it may be difficult to ascertain if a given IgG kappa band in a patient with IgG kappa myeloma is a reflection of the original disease or development of an oligoclonal response. Similar difficulties in interpretation of other immunoglobulin types and light chains also apply $[4,16]$.

The incidence of oligoclonal pattern in plasma cell myeloma patients has a wide range in the literature from single digits to $73 \%[15,17,18]$. The incidence is higher with newer chemotherapeutic agents than with older ones, with autologous stem cell transplantation (ASCT) than without ASCT, and higher yet with allogeneic hematopoietic stem cell transplantation [14]. Occasionally the isotype of the myeloma monoclonal protein may undergo a switch as a result of treatment [19].

In addition to the oligoclonal pattern due to endogenous production of immunoglobulins, extraneous/abnormal monoclonal IgG kappa band may be seen in patients treated with monoclonal antibodies. Treatment with myeloma specific monoclonal antibodies, namely daratumumab and elotuzum$\mathrm{ab}$, is associated with detectable monoclonal IgG kappa in the sera of recipients due to high dose of the therapeutic monoclonal antibodies. Mills and Murray addressed this issue and its mitigation [20-22].

The development of oligoclonal pattern in patients treated with chemotherapy and particularly hematopoietic stem cell transplants has the potential to complicate the interpretation of SPEP/SIFE results and ascertainment of complete response. Oligoclonal bands may also alter the $\kappa / \lambda$ ratio in SFLCA to produce misleading results $[23,24]$.

This retrospective observational study was undertaken to assess the frequency, timing, and immunoglobulin types of oligoclonal bands in plasma cell myeloma patients undergoing ASCT. The impact of the development of oligoclonal pattern on SFLCA and $\kappa / \lambda$ ratio was also assessed. Potential solutions to these issues are also addressed.

\section{Methods}

This retrospective observational study was carried out at a medical school-affiliated, 500-bed tertiary care hospital, in the southeastern USA. The institutional review board approved the study protocol. The laboratory performing the tests is CLIA/CAP accredited.

\section{Protein electrophoresis}

SPEP was performed by agarose gel electrophoresis using Helena SPIFE 3000 system with fractionation into six classic protein zones. Quantitative evaluation for the usual proteins and any M-proteins was performed by scanning the gel at 595 $\mathrm{nm}$ on an EDC densitometer. If the monoclonal protein overlapped a normal protein band, the concentration of the combined peak was reported. SFLCA was performed on Siemens ADVIA 2400 instrument, using Freelite kits and reagents from the Binding Site [12].

\section{Patient and data selection}

The laboratory records for SPEP/SIFE and SFLCA were retrieved for all patients who had either test done between January 2010 and December 2016. Results of any tests done for these patients before 2010 were also recovered so as to have a longitudinal record of the data. The data were pruned to keep only the records of patients with a diagnosis of plasma cell myeloma. Patients with localized plasmacytoma and plasma cell leukemia were excluded. Only the patients in whom at least three SPEP/SIFE test results were available were included. In patients who received ASCT, at least two of the observations needed to be in the post-transplant phase.

Only patients with autologous stem cell transplants were included. One patient with a syngeneic stem cell donor was included in the study group. Recipients of allogeneic stem cells were excluded from the study.

The SPEP/SIFE and SFLCA results were retrieved for the patients meeting the criteria listed above. Not all instances of SPEP/SIFE test had SFLCA data and vice versa. The records of SPEP/SIFE were evaluated for oligoclonal pattern at any time during the course of the patients' illness following the establishment of the diagnosis of plasma cell myeloma. While technically incorrect, even a single protein of restricted heterogeneity different from the primary monoclonal immunoglobu- 
Table 1. Oligoclonal Frequency

\begin{tabular}{lllll}
\hline & No ASCT & Pre-ASCT & ASCT & Post-ASCT \\
\hline Patients & 68 & NA & 159 & NA \\
Observations - N & 816 & 394 & 2,129 & 1,735 \\
Patients with oligo & $6(8.8 \%)$ & 5 & 95 & $92 *(57.9 \%)$ \\
Observations with oligo & 18 & 13 & 348 & 335 \\
\hline
\end{tabular}

In 227 patients the relevant data regarding SPEP/SIFE and SFLCA were available. Autologous hematopoietic stem cell transplantation was carried out in 159 patients. At least three observations were available in all patients and in the ASCT patients, at least two observations were available following stem cell transplantation. The frequencies of oligoclonal pattern in patients without ASCT, prior to ASCT, and following ASCT are given. The incidence of oligoclonal pattern was markedly and significantly higher after ASCT. *Three patients had oligoclonal I pattern before ASCT but not after transplantation. The frequency of oligoclonal pattern was significantly higher following ASCT than that observed without ASCT ( $P=0.000003)$.

lin was characterized as oligoclonal band; however, the term oligoclonal band or pattern will be used instead of abnormal protein band, as the restricted heterogeneity immunoglobulins are not "abnormal".

\section{Results interpretation and retrieval of key data}

The sign-out pathologist usually recorded the location of the malignant monoclonal peak in terms described previously, i.e. location of the band in beta region, overlapping with transferrin or $\mathrm{C} 3$ band, at the beta-gamma interface, in the anodic gamma region, mid-gamma region, cathodic gamma region, or the cathodic end of gamma region $[12,25]$. Having a record of such description facilitated the detection of a new band, especially when it was of the same immunoglobulin type as the primary malignant clone. It is noted that the area of distribution of the immunoglobulin bands is limited to a small area on SPEP and it was not always feasible to ascertain if a given band of the same immunoglobulin type as the primary malignant monoclonal immunoglobulin was part of an oligoclonal response or recurrence or persistence of the malignant clone. In cases of ambiguity, it was not considered to be oligoclonal. The presence of additional light chain bands of the same type as the malignant immunoglobulin was not taken as an indication of oligoclonal pattern. Light chain bands of different types than those seen in the malignant clone were noted to be oligoclonal. Heavy chain bands alone or heavy chain bands with light chains in which either chain was different from that of the malignant clone were judged to be oligoclonal.

Polyclonal response was not recorded as oligoclonal even though it may represent the same process and has the potential to alter the $\kappa / \lambda$ ratio to produce misleading results [11]. Progression of oligoclonal pattern to polyclonal pattern was noted frequently but was not quantified.

The retrospective review facilitated the recognition of oligoclonal bands as the latter were often transient and were missing in later observation.

Serum concentrations of kappa and lambda light chains and $\kappa / \lambda$ ratio were recorded. There were many instances in which either only SPEP/SIFE or only SFLCA results were available.

The time of appearance of the oligoclonal pattern was not- ed, as being before ASCT or its presence in patients who did not receive hematopoietic stem cell transplants. In the posttransplant patients, the occurrence of oligoclonal bands was tagged with time in months following ASCT.

\section{Statistical methods}

Comparison between and among groups was carried out by the Chi-square test.

\section{Results}

A total of 227 patients met the criteria for inclusion in the study. There were 3,134 observations of SPEP/SIFE and/or SFLCA in these patients. SPEP/SIFE data were available in 2,945 of the instances. One hundred fifty-nine of the 227 patients (70\%) received autologous stem cell transplants (Table 1).

In the 68 patients who did not receive stem cell transplantation, only six $(8.8 \%)$ patients exhibited oligoclonal pattern in a total of 18 of $816(2.2 \%)$ observations. The occurrence of oligoclonal pattern at $57.9 \%$ was significantly more frequent in patients with ASCT as compared to patients without ASCT (Table 1).

For the 159 patients that received ASCT, five patients exhibited an oligoclonal pattern in 13 of the 394 observations (3.3\%) before ASCT. In the post-transplant setting, 92 of the $159(57.9 \%)$ patients displayed oligoclonal pattern. The number of observations in patients with ASCT had a far higher incidence of oligoclonal pattern as compared to observations before ASCT or observations in patients without ASCT. The differences remained highly significant when adjusted for the patients and observation of patients who displayed oligoclonal pattern before receiving ASCT versus incidence after ASCT (Tables 1 and 2).

The oligoclonal patterns were segregated by the light chain of the oligoclonal immunoglobulins for the observations in which concomitant SFLCA data were available. Of the 251 such instances, kappa chain only was noted in 44 observations, lambda chain only in 53, and both were present in 154 instances (Table 3). In general, oligoclonal bands with kappa and lambda chains, either light chains alone or with heavy 
Table 2. ASCT Samples and Oligoclonal Frequency

\begin{tabular}{llll} 
& Obs. & Obs. with oligo & \\
\hline Obs. without and before ASCT & 1,210 & $31(2.56 \%)$ & Chi-square: 147.8 \\
Obs. after ASCT & 1,735 & $335(19.31 \%)$ & $\mathrm{P}<<0.00001$ \\
Obs. in pts without ASCT & 816 & $18(2.21 \%)$ & Chi-square: 98.73 \\
Obs. in pts with ASCT & 2,129 & $348(16.35 \%)$ & $\mathrm{P}<<0.00001$ \\
\hline
\end{tabular}

Samples from patients in post-ASCT state had significantly higher frequency of oligoclonal pattern as compared to pre-transplant state and patients who did not receive ASCT. Obs.: observations in samples; Oligo: oligoclonal pattern.

chains, were present in nearly equal numbers, and the bands with kappa chains were generally more prominent, especially on SIFE.

Of the 251 observations identified above, kappa chain was the light chain type of the primary malignant lesion in 167 cases and lambda chain was light chain type in 84 cases. The SFLCA results in these patients were classified according to the criteria and classification, as described earlier [12]. Briefly, if the $\kappa / \lambda$ ratio was greater than 1.65 in a patient with kappa chain malignant lesion, the SFLCA result was classified as concordant; if the ratio was between 0.23 and 1.65 , it was labeled as non-concordant; and if the ratio was less than 0.23 , it was classified as discordant. Similarly for lambda chain lesions, $\kappa / \lambda$ ratio of less than 0.23 was concordant, $0.23-1.65$ was nonconcordant, and greater than 1.65 was classified as discordant. The data for the 251 observations in the post-ASCT period in which both SPEP/SIFE and SFLCA results were available are shown in Table 4 . The discordance rate of $15.5 \%$ was significantly higher for lambda chain primaries with oligoclonal pattern (Table 4). The number of observations with oligoclonal pattern before ASCT or in patients without ASCT for which concomitant SFLCA results were available was not meaningful for statistical analysis.

The timing and frequency of oligoclonal patterns in posttransplant patients is shown in Figure 1. More than half of the oligoclonal patterns developed within the first year following ASCT. The earliest detection of oligoclonal pattern was at 2 months after ASCT and a few instances were noted even after 5 years. It is possible that oligoclonal pattern may have developed before 2 months but SPEP/SIFE was usually not done till more than 1 month after ASCT.

The pattern of progression of oligoclonal pattern in an instructive patient is shown in Figure 2. At presentation (Fig. $2 \mathrm{a}$ ), she had a $4.13 \mathrm{~g} / \mathrm{dL}$ IgG lambda monoclonal protein spike with suppression of background immunoglobulins (February 2010). Following chemotherapy, she received ASCT in September 2010. In November 2010, one prominent IgG kappa

Table 3. Light Chain Type in Oligoclonal Patterns

\begin{tabular}{lllll}
\hline Oligo quality & Kappa & Lambda & Both & Total \\
\hline & 44 & 53 & 154 & 251 \\
\hline
\end{tabular}

For the samples presented in Table 2, the oligoclonal bands with kappa and lambda light chains were about equally distributed. However, judging by the intensity of staining in SIFE, kappa bands tended to be in higher concentration. band, one fainter more cathodic IgG kappa band, and two lambda chain bands were noted, in addition to a trace amount of the original, malignant IgG lambda spike (Fig. 2b). All of the oligoclonal bands were cathodic to the location of the primary malignant clone. The oligoclonal nature of these bands was further validated by their disappearance later in the course of the disease (Fig. 2c). In some patients, a polyclonal pattern was noted in the course of the disease either contemporaneous with the oligoclonal bands or following the disappearance of the oligoclonal bands. In this patient, the $\kappa / \lambda$ ratio varied from lambda dominant to kappa dominant, neutral, and lambda dominant as detailed in Figure 2.

\section{Discussion}

SPEP/SIFE and UPEP/UIFE form the gold standard tests for diagnosing and monitoring monoclonal immunoglobulin disorders [9]. The findings of SPEP/SIFE and UPEP/UIFE are taken along with the bone marrow examination results in diagnosis and monitoring of treatment [4]. Assay for SFLC has been promoted as an adjunct; however, the usefulness of the assay has been questioned [10-12, 25, 26].

With improvements in the treatment of plasma cell myeloma, patients are living longer. Treatment with newer agents and especially with hematopoietic stem cell transplantation has resulted in a measurable incidence of oligoclonal banding in SPEP/SIFE [16, 17, 23, 24, 27, 28]. The implication of oligoclonal pattern as a marker of better outcome is in equipoise as evidence and opinions have been presented suggesting that oligoclonal pattern is a marker of good outcome and others not finding evidence to support favorable outcome in plasma cell myeloma patients with abnormal protein bands or oligoclonal pattern in SPEP/SIFE [16, 17, 23, 24, 27].

The mechanisms underlying the development of oligoclonal bands have also not been settled and it is likely that more than one factor or mechanism is involved. Clonal proliferations of B cells/hematogones (benign precursor B cells) in bone marrow recovering from chemotherapy due to immune reconstitution may result in the development of oligoclonal patterns in serum. The clonal proliferation of transfused hematopoietic stem cells in patients treated with stem cell transplants may also amplify the process. The clonal proliferation may be further amplified in allogeneic stem cell transplants due to graft vs. host reaction. Oligoclonal pattern is also seen in non-myeloma patients in response to infections or autoim- 
Table 4. SFLCA and Light Chain Type of Primary Myeloma Protein Samples

\begin{tabular}{lllll}
\hline \multirow{2}{*}{ Primary LC } & \multicolumn{3}{c}{ SFLCA results } & \\
\cline { 2 - 5 } & Concordant & Non-concordant & Discordant & Chi-square: 41.25 \\
\hline Kappa -167 & 69 & 98 & 0 & $\mathrm{P}<0.00001$ \\
\hline Lambda -84 & 11 & 60 & $13(15.5 \%)$ & \\
\hline
\end{tabular}

For 251 samples with oligoclonal pattern, both the light chain type of the myeloma protein and SFLCA results were available. The results of the concordance of the SFLCA result with that of the malignant light chain revealed $13(15.5 \%)$ cases of lambda light chain type (not just lambda light chain in isolation but any immunoglobulin with lambda light chain) exhibited kappa chain dominant $\mathrm{K} / \lambda$ ratio. It could be surmised that of the 69 samples from patients with kappa chain myeloma protein, about $15.5 \%$ would not have exhibited kappa chain dominance of $\mathrm{K} / \lambda$ ratio without oligoclonal pattern. Thus, the presence of oligoclonal pattern further mars the marginal usefulness of SFLCA.

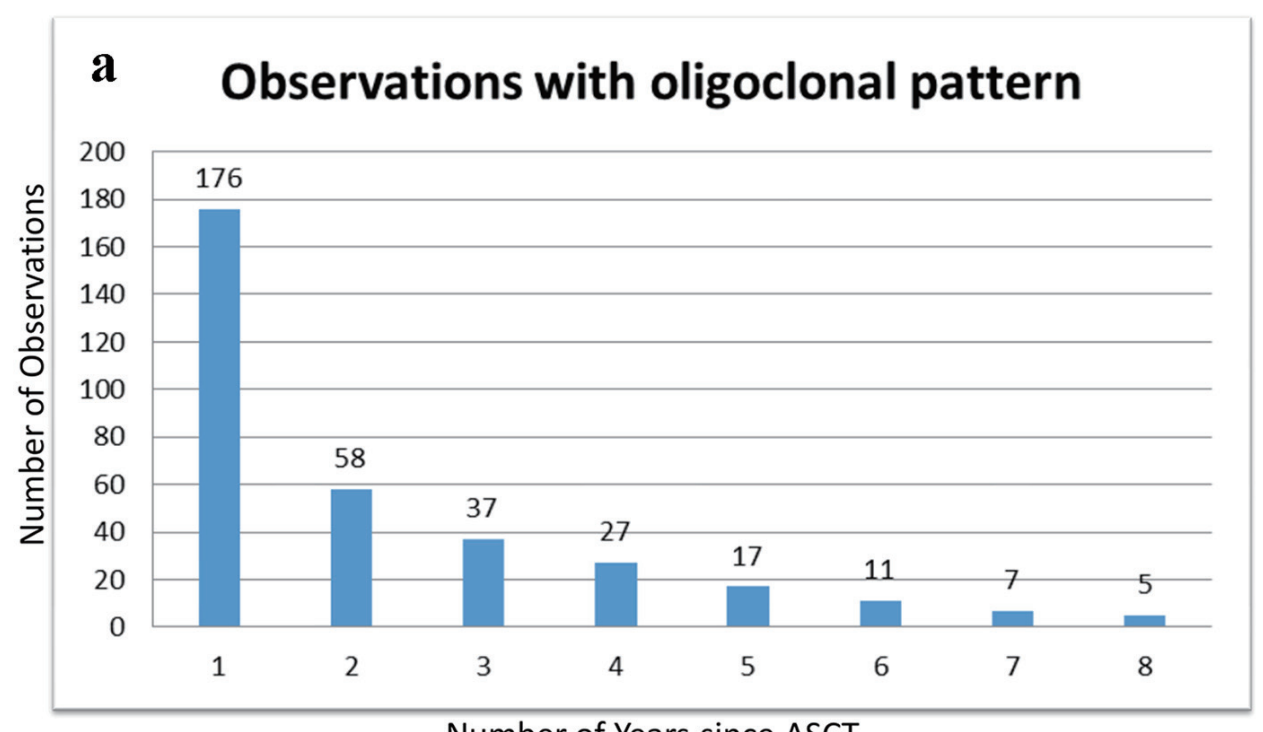

Number of Years since ASCT

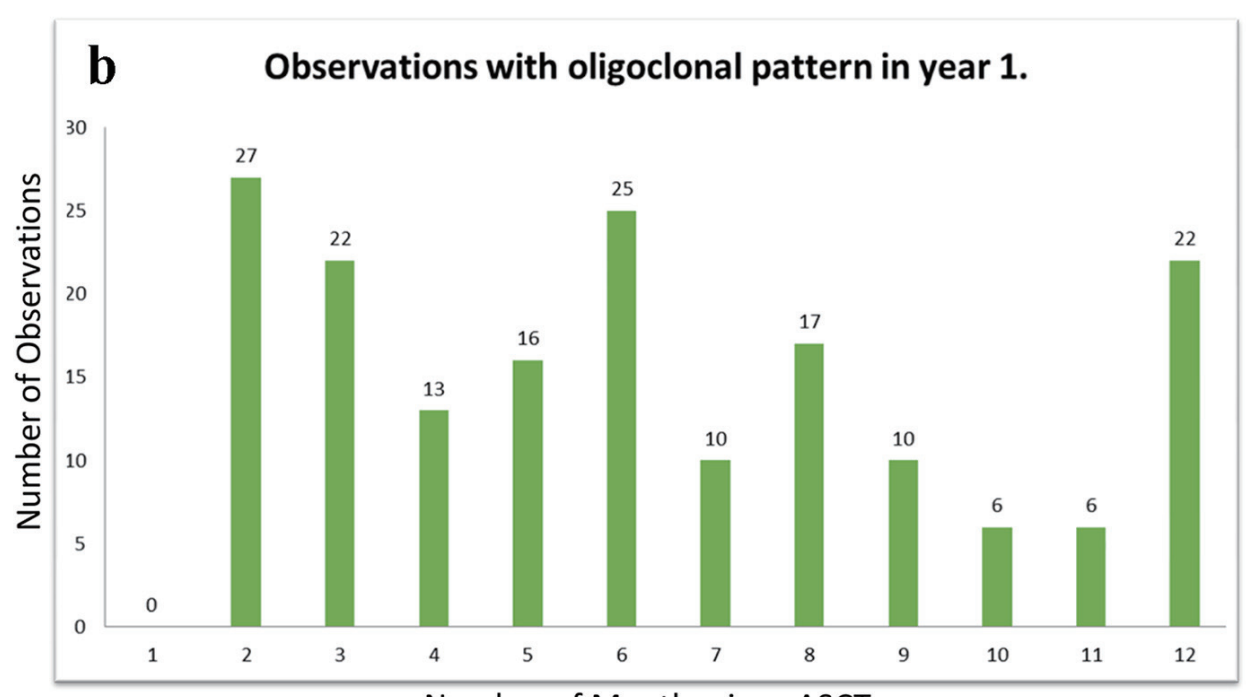

Number of Months since ASCT

Figure 1. $(a, b)$ Chronology of oligoclonal bands. More than half of the 321 instances of oligoclonal bands were noted in the first year after ASCT, though the bands continued to occur up to 8 years after transplantation as shown in chart (a). Chart (b) shows the frequency of oligoclonal bands in the first year, at monthly intervals. No oligoclonal bands were noted in the first month, likely mostly due to paucity of observations during that period. 


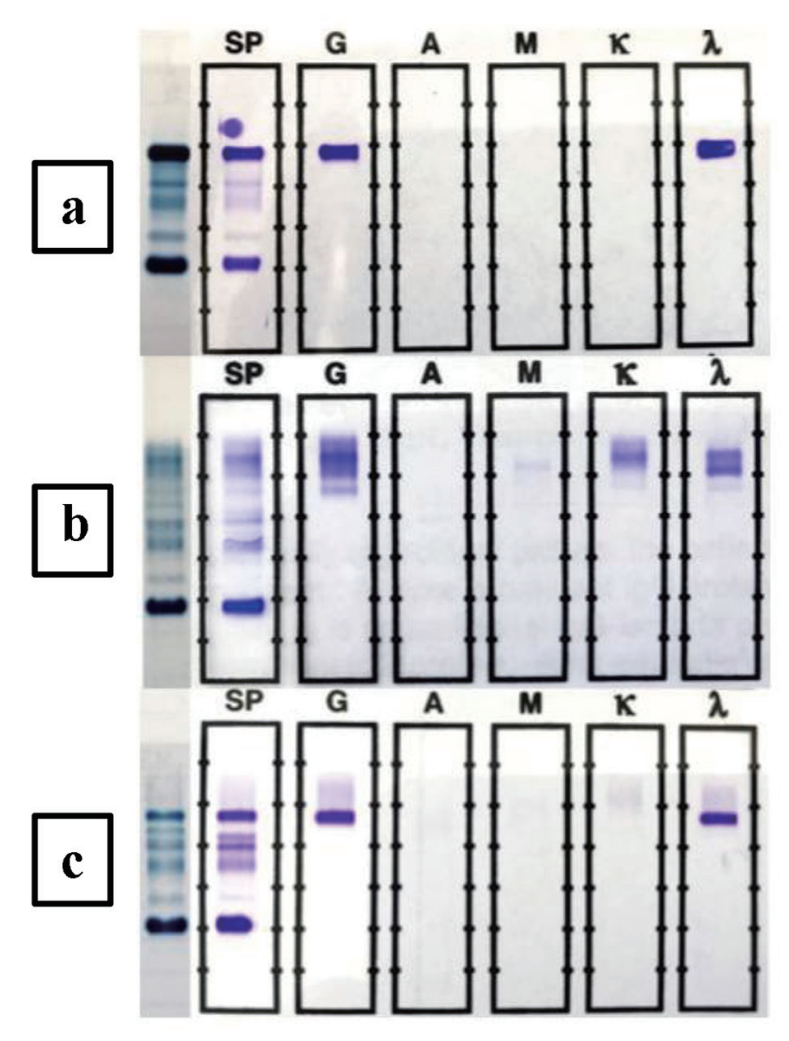

Figure 2. Time course of oligoclonal patterns in a selected patient. The SPEP patterns are shown in the left lane in all of the three SPEP/SIFE records shown. The top SPEP/SIFE (a) was done in February 2010 and displayed a monoclonal IgG lambda band in the anodal gamma region. When the protein was present in high concentration $(4.13 \mathrm{~g} /$ $\mathrm{dL}$ ), as was the case at this time, the band overlapped the $\mathrm{C} 3$ band. The $K / \lambda$ ratio was appropriately lambda dominant at 0.0001 . The middle SPEP/SIFE (b) was done in November 2010, 2 months after ASCT. The original malignant IgG lambda band is detectable in the anodal gamma region. One prominent IgG kappa, an additional fainter IgG kappa band, cathodal to the more prominent one, and two lambda light chain bands are readily detectable, cathodal to the malignant band. Despite the presence of original monoclonal IgG lambda and two oligoclonal lambda bands, the $\mathrm{K} / \lambda$ ratio was kappa dominant at 18.9. The $\mathrm{K} / \lambda$ ratio remained normal/neutral for the next 28 months, despite the recurrence of the malignant IgG lambda. The $k / \lambda$ ratio became lambda dominant when the concentration of the recurrent malignant IgG lambda monoclonal protein reached $1.67 \mathrm{~g} / \mathrm{dL}$. The lower SPEP/SIFE (c) was done in September 2016 and by then all of the oligoclonal bands had disappeared and the malignant IgG lambda band was present at a concentration of $2.62 \mathrm{~g} / \mathrm{dL}$. While it entails the benefit of retrospective review, the two lambda light chain bands depicted in the middle figure did not represent light chain escape in a treated patient but were part of an oligoclonal response.

mune processes and the same may apply to myeloma patients. It has also been suggested that the oligoclonal pattern may reflect autoimmune response to myeloma antigens, especially in allogeneic stem cell transplants. The abnormal protein bands and oligoclonal pattern may be a reflection of an abnormality in the B-cell maturation process [15-17, 28-30].

Irrespective of the mechanism of development of oligoclonal bands, these have a bearing on the interpretation of
SPEP/SIFE and SFLCA results and this issue is the subject of this communication. As has been reported before, polyclonal increase in gamma globulins tends to favor immunoglobulins with kappa light chains and skewing of the $\kappa / \lambda$ ratio in favor of kappa dominance $[8,11,12,23,31]$.

As presented earlier, location of the primary monoclonal spike was recorded in the reports of SPEP/SIFE findings. The location was described as being at the cathodic end, cathodic gamma region, mid-gamma region, anodal gamma region, interface of beta and gamma region, or in beta region and if the band overlapped one of the two proteins in beta region, i.e. transferrin and C3, the overlap location was noted [12, 24, 25]. Only monoclonal IgA protein overlapping the alpha 2 globulin was observed. By comparing of the location of the oligoclonal band to the recorded location of the primary malignant monoclonal immunoglobulin, it was often feasible to ascertain that the oligoclonal band in question was not recurrent malignant immunoglobulin even if it had the same heavy and light chain composition. In this retrospective review, this identification was facilitated by the usually transient nature of the oligoclonal bands. Disappearance of the band, with or without the persistence of the primary monoclonal immunoglobulin, facilitated its recognition as oligoclonal response. If the oligoclonal pattern consisted of immunoglobulin of different composition than the primary malignant clone, its designation as oligoclonal was more straightforward, especially with later disappearance of the clone. Light chain bands, including laddering of light chains, were not considered oligoclonal pattern if the light chain was of the same type as noted in the primary malignant clone.

Even with noting the location of the primary malignant clone, it was often difficult to discern if a given band was in a different location than the primary clone. This highlights the need for a method with higher resolution than the current technology offered by vendors. Capillary electrophoresis and subtraction method of identifying the monoclonal/oligoclonal bands is not likely to be helpful in oligoclonal bands of the same heavy and light chain type as the primary malignant clone. If further studies point to clinical significance of identifying oligoclonal pattern as favorable indicator, it may provide an impetus to the vendors to develop higher resolution electrophoresis methods and equipment. Isoelectric focusing does provide higher resolution; however, this method may not be clinically practical as isoelectric focusing generates multiple bands from clones seen as monoclonal by conventional agarose gel electrophoresis [15, 32].

Oligoclonal banding distorts the results of SFLCA, mostly due to dominance of kappa chain production. This is further complicated by the under-recognition of lambda chains by SFLCA as has been presented earlier [12]. For example, at first encounter, $90 \%$ of the monoclonal gammopathy of undetermined significance (MGUS) lesions with lambda chain monoclonal protein (including those with IgG or other heavy chains) have a falsely normal $\kappa / \lambda$ ratio. The corresponding figures, for lambda chain lesions, of smoldering myeloma and plasma cell myeloma are $60 \%$ and $50 \%$, respectively [12]. The under-recognition of lambda light chains was hypothesized to be due to the greater tendency of lambda chains to aggregate, dimerize, or polymerize, thus making the epitopes recognized the anti- 
body inaccessible to the antibody, resulting in underestimation of free lambda light chains. Even in patients without monoclonal gammopathy, kappa chains tend to dominate. In 292 patients without monoclonal gammopathy, 69 had abnormal kappa/lambda ratio; however, only five of the 69 specimens showed lambda dominance in contrast to 64 of 69 samples showing kappa dominance. This distortion was further magnified in patients with polyclonal gammopathy. In 93 samples with $>1.6 \mathrm{~g} / \mathrm{dL}$ of gamma globulins, 51 displayed an abnormal kappa/lambda ratio; however, only two of the 51 samples had lambda dominance in contrast to kappa dominance in 49 of 51 samples $[11,12]$.

A similar distortion in $\kappa / \lambda$ ratio is noted due to oligoclonal bands. In 251 instances where oligoclonal banding was noted, $\kappa / \lambda$ ratio data were available, as shown in Table 3 . Of the 167 samples in which the primary malignant clone had a kappa light chain, $41 \%$ of the samples had an appropriate, concordant, kappa dominant $\kappa / \lambda$ ratio, with about $59 \%$ having normal, non-concordant, $\kappa / \lambda$ ratio. None of the samples displayed a discordant lambda dominance. However, for the samples in which lambda was the light chain of the primary malignant clone, only $13 \%$ had an appropriate, concordant lambda dominant $\kappa / \lambda$ ratio and $70 \%$ has a non-concordant, normal $\kappa / \lambda$ ratio. It is noteworthy that more samples had a discordant, kappa dominant $\kappa / \lambda$ ratio $(15.5 \%)$ in these lambda chain lesions than the $13 \%$ concordant lambda dominance. It could be concluded that the kappa dominant $\kappa / \lambda$ ratio in these samples with lambda chain primary malignant clone was upended by the excess kappa light chain production by the oligoclonal bands and the likely under-detection of lambda chains.

If the same $15.5 \%$ false rate noted in lambda chain malignant lesions were applied to the kappa chain lesions, it could be construed that about 26 of the 69 concordant kappa dominant $\kappa / \lambda$ ratios were contributed by oligoclonal bands with kappa chains.

Thus the development of oligoclonal bands renders the already dubious usefulness of SFLCA even less meaningful and positively misleading in some (at least $15.5 \%$ ) instances $[11,12,25]$.

More than half of the oligoclonal banding was noted within the first year following ASCT. No oligoclonal bands were noted before 2 months following ASCT, but that may be due, in part, to paucity of observations sooner than 2 months after ASCT. First time development of oligoclonal bands may be delayed to 5 years after ASCT and it becomes critical to recognize this development as a benign process and not a recurrence of the malignant clone. Having higher resolution SPEP/SIFE methods would be useful in identifying the oligoclonal band with same immunoglobulin composition as the primary malignant clone. This is important due to the fact that IgG kappa is the most common immunoglobulin type of plasma cell myelomas and is likely one of the most common immunoglobulin types of oligoclonal bands. As demonstrated in a recent publication, recording the location of the monoclonal spike can serve as a measure to ensure specimen integrity and recognize mislabeled samples [33]. A higher resolution electrophoresis system would enhance this capability.

The occurrence of oligoclonal pattern in status-post chemotherapy patients may interfere with determination of complete response and the same issue arises following ASCT [13-16].

This retrospective observational study has the usual drawbacks of such studies in that the data are not collected in a systematic manner. Ideally a prospective study with collection of sample at regular intervals in all patients would provide a more meaningful result. It is likely that the observed $58 \%$ of patients exhibiting oligoclonal pattern following ASCT, in this study, is an under-estimate due to irregular schedule of testing. The reported $73 \%$ rate of patients having oligoclonal pattern is likely to be closer to the truth and may still be an under-estimate [15]. It is possible that frequent sampling may disclose transient oligoclonal patterns in all patients who receive ASCT. Despite these drawbacks, the findings strongly support noting the location of monoclonal peaks to assist in identifying oligoclonal bands as being different from the malignant clones. The findings also highlight the need for higher resolution electrophoretic methods to obviate the need for using mass-spectrometry for clinical samples [34]. The recognition of oligoclonal pattern as a complicating factor in assessing complete response is recognized and is further highlighted by these results. The previously described shortcomings of the SFLCA are further amplified by the findings and cast more doubt on the clinical usefulness and medical necessity of the serum free light chain assay $[11,12]$.

\section{Conclusions}

Higher resolution protein separation methods are needed to effectively differentiate oligoclonal bands from malignant monoclonal proteins. The methods need to be simple enough for use in a routine clinical laboratory.

Recording of the location of the monoclonal protein spike is useful in differentiation of oligoclonal bands from monoclonal protein, and to ascertain specimen integrity.

Effective differentiation of oligoclonal bands from monoclonal protein may only be possible in retrospect, at the disappearance of the oligoclonal band.

Presence of oligoclonal bands further degrades the performance of SFLCA and $\kappa / \lambda$ ratio and results of SFLCA should not be used in ascertaining complete response or stringent complete response.

\section{Acknowledgments}

Shikhar Vyas, MD, kindly assisted with the illustrations. Review of the manuscript by Natasha Savage, MD and Christine Litwin, MD is gratefully acknowledged. The technical assistance of Ms. Constance Johnson, Ms. Kathy Graham and, Ms. Renee Arthur is acknowledged with gratitude.

\section{References}

1. Kyle RA, Rajkumar SV. Multiple myeloma. Blood. 2008;111(6):2962-2972.

2. Howlader N, Noone AM, Krapcho M, Miller D, Bishop K, Altekruse SF, Kosary CL, Yu M, Ruhl J, Tatalovich Z, Mariotto A, Lewis DR, Chen HS, Feuer EJ, Cronin 
KA (eds). SEER Cancer Statistics Review, 1975-2013, National Cancer Institute. Bethesda, MD, http://seer.cancer.gov/csr/1975_2013/, based on November 2015 SEER data submission, posted to the SEER web site, April 2016.

3. Raab MS, Podar K, Breitkreutz I, Richardson PG, Anderson KC. Multiple myeloma. Lancet. 2009;374(9686):324339.

4. Rajkumar SV, Kumar S. Multiple Myeloma: Diagnosis and Treatment. Mayo Clin Proc. 2016;91(1):101-119.

5. Osterborg A, Mellstedt H. Monoclonal and biclonal immunoglobulin-producing disorders. Eur J Haematol Suppl. 1989;51:11-18.

6. Wang E, Kulbacki E, Stoecker M. Concomitant Waldenstrom macroglobulinemia and IgA plasmablastic myeloma in a patient with untreated IgM paraproteinemia: sequential development of biclonal B-cell neoplasms over a 10-year period in a single individual. Hum Pathol. 2012;43(7):1135-1141.

7. Kyle RA, Robinson RA, Katzmann JA. The clinical aspects of biclonal gammopathies. Review of 57 cases. Am J Med. 1981;71(6):999-1008.

8. Kumar S, Paiva B, Anderson KC, Durie B, Landgren O, Moreau P, Munshi N, et al. International Myeloma Working Group consensus criteria for response and minimal residual disease assessment in multiple myeloma. Lancet Oncol. 2016;17(8):e328-346.

9. Dimopoulos M, Kyle R, Fermand JP, Rajkumar SV, San Miguel J, Chanan-Khan A, Ludwig H, et al. Consensus recommendations for standard investigative workup: report of the International Myeloma Workshop Consensus Panel 3. Blood. 2011;117(18):4701-4705.

10. Bradwell A. Serum Free Light Chain Analysis plus Hevylite, 7 ed. Birmingham, UK: The Binding Site Group Ltd. 2015.

11. Singh G. Serum Free Light Chain Assay and kappa/ lambda Ratio Performance in Patients Without Monoclonal Gammopathies: High False-Positive Rate. Am J Clin Pathol. 2016;146(2):207-214.

12. Singh G. Serum Free Light Chain Assay and kappa/ lambda Ratio: Performance in Patients With Monoclonal Gammopathy-High False Negative Rate for kappa/lambda Ratio. J Clin Med Res. 2017;9(1):46-57.

13. Landgren O, Rajkumar SV. New Developments in Diagnosis, Prognosis, and Assessment of Response in Multiple Myeloma. Clin Cancer Res. 2016;22(22):5428-5433.

14. Fernandez de Larrea C, Tovar N, Cibeira MT, Arostegui JI, Rosinol L, Elena M, Filella X, et al. Emergence of oligoclonal bands in patients with multiple myeloma in complete remission after induction chemotherapy: association with the use of novel agents. Haematologica. 2011;96(1):171-173.

15. Hall SL, Tate J, Gill D, Mollee P. Significance of abnormal protein bands in patients with multiple myeloma following autologous stem cell transplantation. Clin Biochem Rev. 2009;30(3):113-118.

16. Tovar N, de Larrea CF, Arostegui JI, Cibeira MT, Rosinol L, Rovira M, Elena M, et al. Natural history and prognostic impact of oligoclonal humoral response in patients with multiple myeloma after autologous stem cell trans- plantation: long-term results from a single institution. Haematologica. 2013;98(7):1142-1146.

17. Jimenez-Zepeda VH, Reece DE, Trudel S, Franke N, Winter A, Chen C, Tiedemann R, et al. Oligoclonal and monoclonal bands after single autologous stem cell transplant in patients with multiple myeloma: impact on overall survival and progression-free survival. Leuk Lymphoma. 2014;55(10):2284-2289.

18. Tovar N, Fernandez de Larrea C, Pedrosa F, Arostegui JI, Cibeira MT, Rosinol L, Elena M, et al. Differential humoral responses against heat-shock proteins after autologous stem cell transplantation in multiple myeloma. Ann Hematol. 2014;93(1):107-111.

19. Zent CS, Wilson CS, Tricot G, Jagannath S, Siegel D, Desikan KR, Munshi N, et al. Oligoclonal protein bands and Ig isotype switching in multiple myeloma treated with high-dose therapy and hematopoietic cell transplantation. Blood. 1998;91(9):3518-3523.

20. Mills JR, Murray DL. Identification of friend or foe: the laboratory challenge of differentiating M-protein from monoclonal antibody therapies. J Appl Lab Med. 2017;1:421-431.

21. Keren DF. Therapeutic complications: a caveat for Mprotein detection. J Appl Lab Med. 2017;1:342-345.

22. Murata K, McCash SI, Carroll B, Lesokhin AM, Hassoun H, Lendvai N, Korde NS, et al. Treatment of multiple myeloma with monoclonal antibodies and the dilemma of false positive M-spikes in peripheral blood. Clin Biochem. 2016.

23. de Larrea CF, Cibeira MT, Elena M, Arostegui JI, Rosinol L, Rovira M, Filella X, et al. Abnormal serum free light chain ratio in patients with multiple myeloma in complete remission has strong association with the presence of oligoclonal bands: implications for stringent complete remission definition. Blood. 2009;114(24):4954-4956.

24. Jo JC, Yoon DH, Kim S, Lee K, Kang EH, Jang S, Park $\mathrm{CJ}$, et al. Clinical significance of the appearance of abnormal protein band in patients with multiple myeloma. Ann Hematol. 2014;93(3):463-469.

25. Heaton C, Vyas SG, Singh G. Audit of Use and Overuse of Serum Protein Immunofixation Electrophoresis and Serum Free Light Chain Assay in Tertiary Health Care: A Case for Algorithmic Testing to Optimize Laboratory Utilization. Am J Clin Pathol. 2016;145(4):531-537.

26. Alejandre ME, Pavlovsky MA, Remaggi G, Corrado C, Fernandez I, Milone G, Pavlovsky A, et al. Serum free light chains and oligoclonal bands in patients with multiple myeloma and autologous stem cell transplantation. Clin Chem Lab Med. 2012;50(6):1093-1097.

27. Fujisawa M, Seike K, Fukumoto K, Suehara Y, Fukaya M, Sugihara H, Takeuchi M, et al. oligoclonal bands in patients with multiple myeloma: its emergence per se could not be translated to improved survival. Cancer Sci. 2014;105(11):1442-1446

28. Alejandre ME, Madalena LB, Pavlovsky MA, Facio ML, Corrado C, Milone G, Bresciani PD, et al. Oligoclonal bands and immunoglobulin isotype switch during monitoring of patients with multiple myeloma and autologous hematopoietic cell transplantation: a 16-year experience. 
Clin Chem Lab Med. 2010;48(5):727-731.

29. Rahlff J, Trusch M, Haag F, Bacher U, Horst A, Schluter $\mathrm{H}$, Binder M. Antigen-specificity of oligoclonal abnormal protein bands in multiple myeloma after allogeneic stem cell transplantation. Cancer Immunol Immunother. 2012;61(10):1639-1651.

30. McKenna RW, Washington LT, Aquino DB, Picker LJ, Kroft SH. Immunophenotypic analysis of hematogones (B-lymphocyte precursors) in 662 consecutive bone marrow specimens by 4-color flow cytometry. Blood. 2001;98(8):2498-2507.

31. Paiva B, van Dongen JJ, Orfao A. New criteria for response assessment: role of minimal residual disease in multiple myeloma. Blood. 2015;125(20):3059-3068.

32. Henry R, Glegg D. Use of isoelectric focusing to discriminate transient oligoclonal bands from monoclonal protein in treated myeloma. Clin Chem Lab Med. 2016;54(6):985-989.

33. Vyas S, Singh G. Location of monoclonal peak as a tool in checking specimen integrity. Practical Laboratory Medicine.

34. Mills JR, Kohlhagen MC, Dasari S, Vanderboom PM, Kyle RA, Katzmann JA, Willrich MA, et al. Comprehensive Assessment of M-Proteins Using Nanobody Enrichment Coupled to MALDI-TOF Mass Spectrometry. Clin Chem. 2016;62(10):1334-1344. 